\title{
STABILIZATION OF HAMILTONIAN SYSTEMS
}

\author{
A. J. VAN DER SCHAFT \\ Department of Applied Mathematics, Twente University of Technology, P.O. Box 217, 7500 AE Enschede, The \\ Netherlands \\ (Received 10 January 1985; received for publication 29 July 1985)
}

Key words and phrases: Hamiltonian systems, Euler-Lagrange equations, asymptotic stability, Lyapunov theory, output feedback.

\section{HAMILTONIAN SYSTEMS}

IN THIS paper we will be concerned with the stabilization by feedback of Hamiltonian systems. In order to facilitate our discussions (especially when applying Lyapunov's second method) we will restrict ourselves to a particular, although natural, subclass of Hamiltonian systems given in the following way [1].

Let $Q$ be an $n$-dimensional smooth manifold, denoting the configuration space, and let $T^{*} Q$ be the cotangent bundle, denoting the phase space or state space. Furthermore there is a smooth $m$-dimensional output manifold $Y(m \leqslant n)$ and a smooth output map $C: Q \rightarrow Y$. (Smooth will mean $C^{x}$ or $C^{k}$, with $k$ sufficiently big, although we shall restrict ourselves in the second part of Section 2 to analytic data.) For simplicity we take $C$ to be submersive, so rank $d C(q)=m$. We assume that the system on $T^{*} Q$ has an internal energy which is the sum of a kinetic energy $K$ and a potential energy $V$. This means that there exists a Riemannian metric $\langle$,$\rangle on Q$, in local coordinates $\left(q_{1}, \ldots, q_{n}\right)$ for $Q$ given by

$$
\sum_{i, j=1}^{n} g_{i j}(q) d q_{i} \otimes d q_{j},
$$

with $g_{i j}$ smooth functions satisfying $g_{i j}(q)=g_{j i}(q)$, for each $i, j$, and $\left(\mathrm{g}_{i j}(q)\right)>0$ for each $q$. Given local coordinates $\left(q_{1}, \ldots, q_{n}\right)$ for $Q$ we define natural coordinates $\left(q_{1}, \ldots, q_{n}, p_{1}, \ldots\right.$, $\left.p_{n}\right)$ for $T^{*} Q$ by letting $\left(q_{1}, \ldots, q_{n}, p_{1}, \ldots, p_{n}\right)$ correspond to the one-form

$$
\sum_{i=1}^{n} p_{i} d q_{i} \text { on } Q
$$

in the point $\left(q_{1}, \ldots, q_{n}\right) \in Q$. In such natural coordinates the kinetic energy $K$ is then defined by

$$
K(q, p)=\sum_{i, j=1}^{n} g^{i j}(q) p_{i} p_{j}
$$

where $\left(g^{i j}(q)\right)$ is the inverse matrix of $\left(g_{i j}(q)\right)$. Finally the potential energy is defined as a smooth function $V: Q \rightarrow \mathbb{R}$. By letting $\pi: T^{*} Q \rightarrow Q$ denote the canonical projection, and by identifying $V$ with $V \circ \pi$ and $C$ with $C \circ \pi$, we obtain a Hamiltonian system with Hamilton 
function $H(q \cdot p)=K(q \cdot p)+V(q)$ and output map $C: T^{*} Q \rightarrow Y$ given by the equations

$$
\begin{array}{ll}
\dot{q}_{i}=\frac{\partial H}{\partial p_{i}} & \\
\dot{p}_{i}=-\frac{\partial H}{\partial q_{i}}+\sum_{j=1}^{m} u_{j} \frac{\partial C_{i}}{\partial q_{i}} & i=1, \ldots, n \\
y_{i}=C_{j}(q) & j=1, \ldots, m
\end{array}
$$

where $C=\left(C_{1}, \ldots, C_{m}\right)$ in local coordinates $y=\left(y_{1}, \ldots, y_{m}\right)$ for $Y$. We refer to Brockett [2] and van der Schaft $[1,3,4]$ for more information and motivation. The input functions $u=\left(u_{1}\right.$, $\ldots\left(u_{m}\right): \mathbb{R} \rightarrow \mathbb{R}^{m}$ are interpreted in the following way. The manifold $T^{*} Y$, being a cotangent bundle, has natural coordinates $\left(y_{1}, \ldots, y_{m}, u_{1}, \ldots, u_{m}\right)$. Now we let a pair of outputs $y$ and inputs $u$ correspond to a point in $T^{*} Y$ endowed with this coordinate system. So the input $u=$ $\left(u_{1}, \ldots, u_{m}\right)$ denotes an element in the fiber of $T^{*} Y$ above the point $y=\left(y_{1}, \ldots y_{m}\right)$. This allows us to define external force forms, or more or less equivalently static output feedback, as sections of $T^{*} Y$, and to give a coordinate free definition of external work [1, 4]. It may happen that some of the input functions $u_{j}$ in (1.1) have to be constant zero: in this case we speak of a degenerate Hamiltonian system. This means that we cannot use all of the control possibilities which are compatible with the outputs.

The above type of Hamiltonian systems (1.1) occurs frequently. Consider for instance a mechanical system described by a Lagrangian function $L(q, \dot{q})=T(q, \dot{q})-V(q)$, with

$$
T(q, \dot{q})=\frac{1}{2} \sum_{i, j=1}^{n} g_{i j}(q) \dot{q}_{i} \dot{q}_{j}
$$

the kinetic energy. Then the Euler-Lagrange equations including the external forces $u_{i}$ are

$$
\frac{\mathrm{d}}{\mathrm{d} t}\left(\frac{\partial L}{\partial \dot{q}_{i}}\right)-\frac{\partial L}{\partial q_{i}}=u_{i} \quad i=1, \ldots, n .
$$

Denoting $p_{i}=\partial L / \partial \dot{q}_{i}$ we arrive at the equivalent Hamiltonian formulation

$$
\begin{aligned}
& \dot{q}_{i}=\frac{\partial H}{\partial p_{i}} \\
& \dot{p}_{i}=-\frac{\partial H}{\partial q_{i}}+u_{i}
\end{aligned} \quad i=1, \ldots, n
$$

with $H(q, p)=K(q, p)+V(q)$, and

$$
K(q, p)=\frac{1}{2} \sum_{i, j=1}^{n} g^{i j}(q) p_{i} p_{j}
$$

Adjoining the output equations

$$
y_{i}=q_{i} \quad i=1, \ldots, n
$$

to (1.3a) we again obtained a system of the form (1.1). In fact given a system (1.1) and given a coordinate system $\left(y_{1}, \ldots, y_{m}\right)$ for $Y$ we can always find, since $C$ is submersive, a coordinate 
system $\left(q_{1}, \ldots, q_{n}\right)$ for $Q$, such that $C_{i}\left(q_{i}, \ldots, q_{n}\right)=q_{i}, i=1, \ldots, m[5]$. Hence we obtain equations (1.3) with a number of external forces or outputs which is smaller than the number of configuration space coordinates (generalized coordinates).

For studying the structural properties of (1.1) or (1.3) we introduce the following Lie algebra $[1,4,6]$. For two functions $F, G: T^{*} Q \rightarrow \mathbb{R}$ the Poisson bracket is defined as

$$
\{F, G\}=\sum_{i=1}^{n}\left(\frac{\partial F}{\partial p_{i}} \frac{\partial G}{\partial q_{i}}-\frac{\partial F}{\partial q_{i}} \frac{\partial G}{\partial p_{i}}\right) .
$$

Let now $\mathscr{C}$ be the smallest subspace of the linear space $C^{*}\left(T^{*} Q\right)$ of smooth functions on $T^{*} Q$ that contains the functions $C_{1}, \ldots, C_{m}$ and is closed w.r.t. Poisson bracketing with $H$ and $C_{1}$, $\ldots, C_{m}$, i.e. if $G \in \mathscr{b}$ then $\{H, G\} \in \mathscr{b}$ and $\left\{C_{i}, G\right\} \in \mathscr{b}$. It is easily seen that $\mathscr{b}$ is actually a Lie algebra w.r.t. the Poisson bracket: if $G_{1}, G_{2} \in \mathscr{C}$, then $\left\{G_{1}, G_{2}\right\} \in \mathscr{\ell}$. The following basic result holds [6].

Theorem 1.1. Consider a Hamiltonian system (1.1) with Lie algebra $\mathscr{~}$. Let $\mathrm{d} \mathscr{G}(x)=$

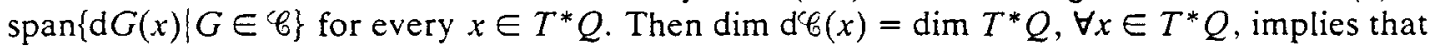
the system (1.1) is strongly accessible as well as locally weakly observable. Conversely, if (1.3) is strongly accessible or locally weakly observable, then $\operatorname{dim} \operatorname{d} \ell(x)=\operatorname{dim} T^{*} Q$ for every $x$ in an open and dense subset of $T^{*} Q$.

The above theorem holds in fact for general Hamiltonian systems. In the case of Hamiltonian systems of the form (1.1) $\mathscr{b}$ has a more refined structure. Define $\mathscr{G}_{0}$ as the linear subspace of $C^{\infty}\left(T^{*} Q\right)$ generated by taking only Poisson brackets with $H$, i.e.

$$
C_{0}=\operatorname{span}\left\{C_{i},\left\{H, C_{i}\right\},\left\{H,\left\{H, C_{i}\right\}\right\}, \ldots, i=1, \ldots, m\right\} .
$$

It is clear that $\mathscr{b}_{0} \subset \mathscr{b}$ and $\overline{\mathscr{C}}_{0}=\mathscr{\ell}$, where ${ }^{-}$denotes the closure w.r.t. Poisson bracketing. It now easily follows from the structure of $H=K+V$ and $C_{i}$ that the expressions in $\mathscr{G}_{0}$ and also in $\mathscr{C}$ are polynomials in the variables $p_{1}, \ldots, p_{n}$ with coefficients which are smooth functions of $\left(q_{i}, \ldots, q_{n}\right)$. In fact every element in $\mathscr{\ell}$ can be written as a sum of homogeneous polynomials in $\left(p_{1}, \ldots, p_{n}\right)$ which are all of even degree, or all of odd degree. (A polynomial $G(q, p)$ in $\left(p_{1}, \ldots, p_{n}\right)$ is homogeneous of degree $k$ if $\left.G\left(q, \lambda p_{1}, \ldots, \lambda p_{n}\right)=\lambda^{k} G\left(q, p_{1}, \ldots, p_{n}\right), \forall \lambda \in \mathbb{R}.\right)$ This special structure of $\mathscr{b}$ has already one important consequence, namely that $\mathscr{b}$ is invariant under the canonical involution $\phi: T^{*} Q \rightarrow T^{*} Q$ given by $\phi(q, p)=(q,-p)$. This implies that every system (1.1) is time-reversible, i.e. if $(u(t), y(t)), t \in \mathbb{R}$, is a possible input-output behavior of the system, then so is the pair $(\bar{u}(t), \bar{y}(t)), t \in \mathbb{R}$, with $\bar{u}(t)=u(-t)$ and $\bar{y}=y(-t)$ (cf. $[1,4]$ ). (Remark: the study of symmetries of $(1.1)$ can also be performed within the framework of the Lie algebra $\mathscr{b}$ [7]. It would be interesting to exploit the structure of $b$ in this context.)

Finally we will enlarge the framework of (1.1), by assuming that we can also measure the derivatives of the output functions, i.e. (some of) the generalized velocities. Let $y_{i}=C_{i}(q)$ be an output of (1.1). Then

$$
\dot{y}_{i}=\left\{H-\sum_{j=1}^{m} u_{j} C_{j}, C_{i}\right\}=\left\{H, C_{i}\right\}-\sum_{j=1}^{m} u_{j}\left\{C_{j}, C_{i}\right\}=\left\{H, C_{i}\right\}
$$


for every $u$. We call the system

$$
\begin{aligned}
& \dot{q}_{i}=\frac{\partial H}{\partial p_{i}} \\
& \dot{p}_{i}=-\frac{\partial H}{\partial q_{i}}+\sum_{j=1}^{m} u_{j} \frac{\partial C_{i}}{\partial q_{i}}
\end{aligned} \quad i=1 \ldots, n
$$

with outputs

$$
\begin{aligned}
& y_{i}=C_{i}(q) \\
& \dot{y}_{i}=\left\{H, C_{i}\right\}(q, p)
\end{aligned}
$$

a Hamiltonian system with extended measurements. Notice that the system (1.6) is locally weakly observable if and only if the original system (1.1) is locally weakly observable, since $\left\{H, C_{i}\right\} \in \mathscr{b}, i=1, \ldots, m$.

\section{STABILIZATION}

Consider a general nonlinear system

$$
\dot{x}=f(x, u), \quad x \in M
$$

with equilibrium point $(x, u)=(0,0)$, i.e. $f(0,0)=0$. It is well known that we can locally construct a feedback $u=\alpha(x)$, with $\alpha(0)=0$, such that $x=0$ is a (locally) asymptotically stable point for $\dot{x}=f(x, \alpha(x)$ ) (we say that $\dot{x}=f(x, u)$ can be stabilized by feedback), if the linearized system $\dot{x}=A x+B u$ with

$$
A=\frac{\partial f}{\partial x}(0,0), \quad B=\frac{\partial f}{\partial u}(0,0)
$$

is controllable [8]. We refer to this method as stabilization by Lyapunov's first method (linearization). Furthermore we remark that his condition is invariant under feedback $u=\alpha(x, \bar{u})$, with $\alpha(0,0)=0$ and $(\partial x / \partial \tilde{u})$ of full rank ( $\bar{u}$ is the new input vector). In fact, denote the feedback transformed system by $\dot{x}=\bar{f}(x, \bar{u})$, with $\bar{f}(x, \bar{u}):=f(x, \alpha(x, \bar{u}))$. Then

and

$$
\begin{aligned}
\tilde{A}=\frac{\partial \tilde{f}}{\partial x}(0,0) & -\frac{\partial f}{\partial x}(0,0)+\frac{\partial f}{\partial u}(0,0) \frac{\partial \alpha}{\partial x}(0,0) \\
& =A+B \frac{\partial \alpha}{\partial x}(0,0)
\end{aligned}
$$

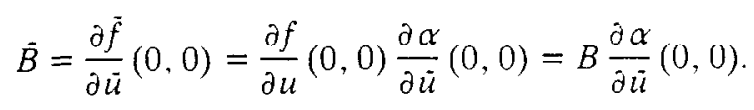

It is clear that $(A, B)$ is controllable if and only if $(\bar{A}, \bar{B})$ is controllable.

Let us for simplicity assume that $f$ is of the form

$$
f(x, u)=A(x)+\sum_{i=1}^{m} u_{i} B_{i}(x)
$$


then the above condition can be equivalently stated in a more geometric way as follows [9]. Define $\mathscr{L}_{0}$ as the following linear subspace of $V^{x}(M)$, the linear space of vector fields on $M$ :

$$
\mathscr{L}_{0}=\operatorname{span}\left\{B_{i},\left[A, B_{i}\right],\left[A,\left[A, B_{i}\right]\right], \ldots, i=1, \ldots, m\right\}
$$

with $[$,$] the Lie bracket. It is then easily seen that, since A(0)=0$, controllability of

$$
\left(\frac{\partial f}{\partial x}(0,0), \frac{\partial f}{\partial u}(0,0)\right)
$$

is equivalent to the condition $\operatorname{dim} \mathscr{L}_{0}(0)=\operatorname{dim} M$, where $\mathscr{L}_{0}(0)=\operatorname{span}\left\{X(0) \mid X \in \mathscr{L}_{0}\right\}$. This condition is customarily referred to as the "ad-condition".

For Hamiltonian systems (1.1) the above condition for stabilization by Lyapunov's first method can be translated as follows. Let $(q, p)=(0,0)$ be an equilibrium point for $(1.1)$, so $(\partial V / \partial q)(0)=0$. Define

and

$$
Q=\left(\frac{\partial^{2} V}{\partial q_{i} \partial q_{j}}(0)\right), \quad P=\left(\frac{\partial^{2} K}{\partial p_{i} \partial p_{j}}(0,0)\right)=\left(g^{i j}(0)\right),
$$

$$
c_{i}=\left(\frac{\partial C_{i}}{\partial q}(0)\right)
$$

Then the system can be made (locally) asymptotically stable around $(q, p)=(0,0)$ if the linear Hamiltonian system

$$
\left(\begin{array}{l}
\dot{q} \\
\dot{p}
\end{array}\right)=\left(\begin{array}{cc}
0 & P \\
-Q & 0
\end{array}\right)\left(\begin{array}{l}
q \\
p
\end{array}\right)+\left(\begin{array}{l}
0 \\
C^{T}
\end{array}\right) u, \quad y=C q
$$

with $c_{i}$ the $i$ th row of the matrix $C$, is controllable. This is also easily seen to be equivalent to the controllability of the pair $\left(P Q, P C^{T}\right)$. The equivalent ad-condition in this case can be expressed by requiring that $\operatorname{dim} \mathrm{d}_{0}(0,0)=\operatorname{dim} T^{*} Q$, where $\mathscr{G}_{0}$ is the subspace of $C^{*}\left(T^{*} Q\right)$ defined in (1.5).

\section{Stabilization of Hamiltonian systems by Lyapunov's second method}

In the case of Hamiltonian systems (1.1) a certainly more natural approach to stabilization is provided by Lyapunov's second or direct method, as noted already by several authors [e.g. Tsinias and Kalouptsidis [10], Jonckheere [11], Marino [12]]. The reason is of course that there is a natural candidate for the Lyapunov function, namely the internal energy $H$ itself, since $(\mathrm{d} / \mathrm{d} t) H=0$ along any trajectory of the system (1.1) with $u=0$. If $H$ possesses a strict local minimum in $(q, p)=(0,0)$ this already implies that the system (1.1) with $u=0$ is (locally) stable (however not asymptotically stable).

Remark. Of course an important advantage of Lyapunov's second method in contrast with Lyapunov's first method is that we are not restricted to the investigation of local (asymptotic) stability, where "local" can mean an arbitrary small neighborhood. Although we shall confine ourselves in the sequel to local (asymptotic) stability, the results can therefore be easily extended to cover the global case as well, using standard Lyapunov theory (cf. La Salle and Lefschetz [13]). 
In the rest of this section we assume that all the data of the Hamiltonian system (1.1) are (real) analytic. We will derive the following main theorem:

THEOREM 2.1. Consider an analytic Hamiltonian system (1.1) with equilibrium point $(q, p)=$ $(0,0)$ and $C_{i}(0)=0, i=1, \ldots, m$. For an analytic function $P: Y \rightarrow \mathbb{R}$ denote $V_{p}=V+P \circ C$. Let $\mathscr{C}_{0}^{P}$ correspond to $H_{P}=V_{P}+K$ and $C_{i}$ as in (1.5). Make now the following assumption: assume that for any $P$ with $P(0)=\mathrm{d} P(0)=0$ the dimension of $\mathrm{d}^{\prime} G_{0}^{P}(q, p)$ is constant for any point $(q, p)$, with $q \neq 0$, in a neighborhood $U$ of $(0,0)$. Then the system can be made locally asymptotically stable around $(0,0)$ using feedback $u=\alpha(q, p)$ if the following two conditions are satisfied.

(a) There exists an analytic function $S: Y \rightarrow \mathbb{R}$ such that $V_{S}(q)=V(q)+S \circ C(q)$ has a strict local minimum in $q=0$ and $\mathrm{d} V_{S}(q) \neq 0$ for every $(q, p) \in U$ with $q \neq 0$.

(b) $\operatorname{dim} \mathrm{d}^{C} b_{0}(q, p)=2 n$ for every $(q, p) \in U$ with $q \neq 0$.

Moreover if both conditions are satisfied then the system can be stabilized using a decentralized output feedback for the Hamiltonian system with extended measurements (1.6) of the form

$$
u_{i}=-k_{i} y_{i}-c_{i} \dot{y}_{i}, \quad i=1, \ldots, m
$$

with $k_{i}$ and $c_{i}$ nonnegative constants.

Proof. The first step is to apply static output feedback (w.r.t. (1.1))

$$
u_{i}=-\frac{\partial S}{\partial y_{i}}(y)+v_{i}, \quad i=1, \ldots, m
$$

with $v=\left(v_{1}, \ldots, v_{m}\right)$ the new inputs. This results in

$$
\begin{aligned}
& \dot{q}_{i}=\frac{\partial H}{\partial p_{i}} \\
& \dot{p}_{i}=-\frac{\partial H}{\partial q_{i}}-\sum_{i=1}^{m} \frac{\partial S}{\partial y_{j}}(C(q)) \frac{\partial C_{j}}{\partial q_{i}}+\sum_{j=1}^{m} v_{j} \frac{\partial C_{j}}{\partial q_{i}}
\end{aligned}
$$

which is a Hamiltonian system with kinetic energy $K$ and potential energy $V_{S}(q)=$ $V(q)+S \circ C(q)$. Since $S$ satisfies condition a the function $V_{S}(q)$ has a strict local minimum in $q=0$ and hence the system (2.8) with $v=0$ is locally stable. Now it is easy to see that there exist nonnegative constants $k_{1}, \ldots, k_{m}$ such that also

$$
\bar{V}(q)=V(q)+\sum_{j=1}^{m} k_{j} y_{j}^{2}
$$

has a strict local minimum in 0 . In fact since $C$ is submersive there exist coordinates $q=\left(q_{1}\right.$, $\left.\ldots, q_{n}\right)$ around 0 for $Q$ such that $C_{j}\left(q_{1}, \ldots, q_{n}\right)=q_{j}, j=1, \ldots, m$. Hence $S \circ C\left(q_{1}, \ldots\right.$, $\left.q_{n}\right)=S\left(q_{1}, \ldots, q_{m}\right)$. Since $V\left(q_{1}, \ldots, q_{n}\right)+S\left(q_{1}, \ldots, q_{m}\right)$ has a local minimum in $q=0$ and $\mathrm{d} V(0)=0$ it follows that $\mathrm{d} S(0)=0$. Now we can choose an $m \times m$ diagonal matrix $D=\operatorname{diag}$ $\left(k_{1}, \ldots, k_{m}\right)$ such that

$$
D>\left(\frac{\partial^{2} S}{\partial q_{i} \partial q_{j}}(0)\right) \quad i, j=1, \ldots, m .
$$


Then in a neighborhood of 0 ,

$$
\dot{V}(q)=V(q)+\sum_{j=1}^{m} k_{j} q_{j}^{2}>V\left(q_{1}, \ldots, q_{n}\right)+S\left(q_{1} \ldots, q_{m}\right)-S(0)>V(0)
$$

for every $\left(q_{1}, \ldots, q_{n}\right) \neq(0, \ldots, 0)$. Hence $\tilde{V}(q)$ possesses a strict local minimum in $q=0$. Furthermore it is easily seen that if $\mathrm{d} V_{S}(q) \neq 0$ for $q \neq 0$ in a neighborhood of 0 , then also $\mathrm{d} \bar{V}(q) \neq 0$ for $q \neq 0$ in a (possibly different) neighborhood of 0 . Therefore instead of applying the feedback (2.7) we may also apply the decentralized output feedback

$$
u_{i}=-k_{i} y_{i}+v_{i} \quad i=1 \ldots m
$$

resulting in a (locally) stable Hamiltonian system with potential energy $\bar{V}$. The second step is to apply the output feedback

$$
v_{i}=-c_{i} \dot{y}_{i}, c_{i}>0, \quad i=1 \ldots, m \text {. }
$$

An easy calculation shows that

$$
\frac{\mathrm{d}}{\mathrm{d} t}(K+\tilde{V})=-\sum_{j=1}^{m} c_{j} i_{i}
$$

where we differentiate along the trajectories of the system (1.1) with $u_{i}=-k_{i} y_{i}-c_{i} \dot{y}_{i}$. Hence it follows from Lyapunov stability theory [13] that the system with the above feedback (2.6) converges in a neighborhood of $(0,0)$ to a maximal invariant subset contained in $E=$ $\left\{(q, p) \mid \dot{y}_{1}(q, p)=\ldots=\dot{y}_{m}(q, p)=0\right\}$. Consider a trajectory of $(1.1)$ with feedback $(2.6)$ contained in $E$. Then $\dot{y}_{j}(q, p)=0, j=1, \ldots, m$, along this trajectory, and hence the inputs $v_{i}=$ $-c_{i} \dot{y}_{i}$ are all zero and the outputs $y_{i}=C_{i}(q)$ are all constant. i.e. $y_{i}=C_{i}(q)=\alpha_{i} . i=1, \ldots$ $m$. Denote the original Hamiltonian system (1.1) with Hamilton function $H$ and $u=0$ by $\Sigma$, and denote the output feedback transformed Hamiltonian system with Hamilton function $\bar{H}=$ $K+\bar{V}$ and $v=0$ by $\bar{\Sigma}$. Let $\mathscr{C}_{0}$ and $\overline{\mathscr{B}}_{0}$ as in (1.5) correspond to $\Sigma$, respectively $\bar{\Sigma}$.

So we have to deal with trajectories of $\dot{\Sigma}$ which are contained in sets $C_{i}(q)=\alpha_{i}, i=1, \ldots$, $m$. For every $x$ on such a trajectory it follows that

$$
\left\{\tilde{H}, C_{i}\right\}(x)=\left\{\tilde{H},\left\{\tilde{H}, C_{i}\right\}\right\}(x)=\ldots=0 .
$$

Hence in particular the trajectories of $\bar{\Sigma}$ contained in $C_{i}(q)=0, i=1, \ldots, m$. are contained in the analytic set

$$
\bar{N}=\left\{(q, p) \mid f(q, p)=0, \forall f \in \bar{\ell}_{0}\right\} .
$$

Moreover, since $(\partial \bar{V} / \partial q)(0)=0$ and $C_{i}(0)=0$, it follows that at least $(0,0) \in \bar{N}$. In the same way it follows that the trajectories of $\Sigma$ contained in $C_{i}(q)=0$ are contained in $N=$ $\left\{(q, p) \mid f(q, p)=0, \forall f \in^{\prime} b_{0}\right\}$ and that $(0,0) \in N$. Now, since $k_{i} y_{i}=0$ on the set $C_{i}(q)=0, i=$ $1, \ldots, m$, the trajectories of $\bar{\Sigma}$ and $\Sigma$ contained in $C_{i}(q)=0$ are actually the same! For using condition (b) we need the following lemma.

LEMMA 2.2 Take the assumption of theorem 2.1. Then: $\operatorname{dim} \mathrm{d}^{\prime} \ell_{0}(q, p)=2 n, \forall(q, p) \in U$ with $q \neq 0$ and $C(q)=0\left\langle\Rightarrow\right.$ there are no trajectories of $\Sigma$ contained in $U$ and the set $C_{i}(q)=0$, $i=1, \ldots, m$, except the origin $(0,0)$. 
Proof. Everything will be in an arbitrary small neighborhood of $(0,0) .(\Rightarrow)$. Take an arbitrary point $(q, p)$ with $q \neq 0$ and $C_{i}(q)=0$. Since $\operatorname{dim} \mathrm{d} G_{0}(q, p)=2 n$ there exists a neighborhood $V$ of $(q, p)$ such that $N \cap V=(q, p)$. Therefore the trajectory passing through $(q, p)$ should be the point $(q, p)$ itself. Now suppose $p \neq 0$. Since $\left(g^{i j}(q)\right)>0$ it follows that $\dot{q} \neq 0$, and we have a contradiction. Furthermore if $p=0$ then $\dot{p}=-(\partial V / \partial q)(q) \neq 0$, since $q \neq 0$. Hence $p(t) \neq 0$ and $\dot{q}(t) \neq 0$ for some $t$, a contradiction. Finally we consider a point $(0, p)$, with $p \neq 0$. Then also $\dot{q} \neq 0$ and we again obtain a contradiction, $(\Leftarrow)$. Suppose dim $\mathrm{d} \mathscr{G}_{0}(q, p)=k<2 n, \forall(q, p)$ with $q \neq 0$ and $C(q)=0$. (Here we use the assumption of theorem 2.1.) Then $M=N \cap\{(q, p) \mid q \neq 0\}$ is an analytic submanifold of dimension $2 n-k$. It is easily seen that $(0,0) \in \bar{M}$ ( denotes closure). Now take a point $(q, p) \in M$. Then $f(q, p)=0$ and $\{H, f\}(q, p)=0$ for every $f \in \mathscr{C}_{0}$. Hence, by analyticity, $X^{r}(q, p) \in M$ for every small $t \geqslant 0$ ( $X_{H}^{t}$ is the integral flow of $\Sigma$ ). Therefore $M$ and hence $N$ contains trajectories of $\Sigma$ different from the origin.

Proof of theorem 2.1 continued. Let now condition (b) be satisfied. Then by lemma 2.2 it follows that $\Sigma$ and hence $\bar{\Sigma}$ do not have trajectories contained in $C_{i}(q)=0$, except $(0,0)$. Hence by lemma 2.2 also $\operatorname{dim} \mathrm{d} \tilde{\mathscr{C}}_{0}(q, p)=2 n$ for every $(q, p) \in U$ with $q \neq 0$ and $C(q)=0$. By the assumption of theorem 2.1 this yields $\operatorname{dim} \mathrm{d} \tilde{\mathscr{C}}_{0}(q, p)=2 n$ for every $(q, p)$ with $q \neq 0$ in a neighborhood of $(0,0)$. Then by the same argument of lemma $2.2(\Rightarrow)$ it follow's that there exist no trajectories of $\bar{\Sigma}$ contained in $C_{i}(q)=\alpha_{i}, i=1, \ldots, m$, except $(0,0)$ if $\alpha_{i}=0$. Hence by La Salle's theorem the origin $(0,0)$ is locally asymptotically stable for the system $(1.1)$ with fecdback (2.6).

Remark 1. Intuitively the fcedback scheme of theorem 2.1 is very clear. First we shape the potential function $V$ to a function with a strict minimum in $q=0$ by applying output feedback $u_{i}=-\left(\partial S / \partial y_{i}\right)+v_{i}$, or $u_{i}=-k_{i} y_{i}+v_{i}$. In fact the most general (state) feedback $u+\alpha(q, p, v)$ under which the system remains Hamiltonian is necessarily of this form $u_{i}=-\left(\partial S / \partial y_{i}\right)+v_{i}$ (van der Schaft [7]). After this output feedback the system is already stable. As a second step we add damping to the system via the feedback $v_{i}=-c_{i} \dot{y}_{i}$, resulting in asymptotic stability. This same rough idea for stabilization can be also found in [10-14]. Related is theorem 2 in Jurdjevic and Quinn [9]. It follows from theorem 2.1 that we do not have to add damping with respect to all generalized coordinates in order to achieve asymptotic stability, but only with respect to a set of functions $C_{1}, \ldots, C_{m}: Q \rightarrow \mathbb{R}$ which is big enough for condition (b) to be satisfied. This is also known from engineering as noted by Jonckheere (1981); in general one local feedback $u_{j}=-c_{j} \dot{q}_{j}$, $j$ fixed, has the tendency to spread all over the system. If $m=n$, i.e. if we observe all generalized coordinates, then conditions (a) and (b) are trivially satisfied (notice that the $2 n$ functions $C_{i},\left\{H, C_{i}\right\}$ are independent), and hence stabilization by Lyapunov's second method is always possible as shown in $[11,14]$.

Remark 2. Condition (b) can be somewhat sharpened. If $F: T^{*} Q \rightarrow \mathbb{R}$ is a first integral of $\Sigma$ (a conserved quantity), then $F$ may be added to the set $\mathscr{b}_{0}$, since $F$ is constant along any trajectory if $\Sigma$. In particular we may always add $H$ to $b_{0}$ in condition (b), since $(\mathrm{d} / \mathrm{d} t) H=0$.

We now wish to make some comments about conditions (a) and (b). Firstly we notice that if condition (a) is satisfied we have to choose the constants $k_{1}, \ldots, k_{m}$ in $(2.6)$ in such a way 
that

$$
\left(\frac{\partial C}{\partial q}(0)\right)^{T} \operatorname{diag}\left(k_{1}, \ldots, k_{m}\right)\left(\frac{\partial C}{\partial q}(0)\right) \geqslant-\left(\frac{\partial^{2} V}{\partial q_{i} \partial q_{j}}(0)\right) .
$$

In fact since $V+S$ has a strict local minimum in 0 ,

$$
\left(\frac{\partial^{2}(V+S \circ C)}{\partial q_{i} \partial q_{i}}(0)\right) \geqslant 0
$$

and hence

$$
\left(\frac{\partial C}{\partial q}(0)\right)^{T}\left(\frac{\partial^{2} S}{\partial y_{i} \partial y_{j}}(C(0))\right)\left(\frac{\partial C}{\partial q}(0)\right) \geqslant-\left(\frac{\partial^{2} V}{\partial q_{i} \partial q_{j}}(0)\right) .
$$

Of course condition (a) as it stands is not very constructive. The following conditions are sufficient (but not necessary) in order that condition (a) is satisfied.

Proposition 2.3. Let the matrix

$$
\left(\frac{\partial^{2} V}{\partial q_{i} \partial q_{j}}(0)\right)
$$

be positive definite restricted to the subspace

Then condition $a$ is satisfied.

$$
\operatorname{Ker}\left(\frac{\partial C}{\partial q}(0)\right)
$$

The proof requires the following simple lemma.

LEMMA 2.4. Let $Q$ be an $n \times n$ symmetric matrix and let $C$ be a surjective $m \times m$ matrix. Then there exists an $m \times m$ symmetric matrix $H$ such that $Q+C^{T} H C>0$ if and only if $Q$ restricted to $\operatorname{Ker} C$ is positive definite.

Proof. (cf. Jongen et al. [15]). The "only if" directions is clcar. Let now $Q \mid \operatorname{Ker} C>0$. Let $W$ be an $n \times(n-m)$ matrix whose columns span $\operatorname{Ker} C$, and let $V$ be an $n \times m$ matrix whose columns span the orthogonal complement of $Q(\operatorname{Ker} C)$. First we prove that the $n \times n$ matrix $(V: W)$ is nonsingular. Let $V \alpha+W \beta=0$, with $\alpha \in \mathbb{R}^{m}$ and $\beta \in \mathbb{R}^{n-m}$. Then $0=$ $W^{T} Q(V \alpha+W \beta)=W^{T} Q W \beta$. Since $Q \mid \operatorname{Ker} C>0$ this yields $\beta=0$ and hence $\alpha=0$. It is easy to see that

$$
(V: W)^{T}\left(Q+C^{T} H C\right)(V: W)=\left(\begin{array}{c:c}
V^{T} Q V+V^{T} C^{T} H C V & 0 \\
\hdashline 0 & W^{T} Q W
\end{array}\right)
$$

Since rank $V^{T} C^{T} H C V=\operatorname{rank}(V: W)^{T} C^{T} H C(V: W)=\operatorname{rank} C^{T} H C$ it follows that $Q+C^{T} H C$ can be made positive by choosing an appropriate $H=H^{T}$.

Proof of proposition 2.3. Apply lemma 2.4 to

$$
Q=\left(\frac{\partial^{2} V}{\partial q_{i} \partial q_{j}}(0)\right) \text { and } C=\left(\frac{\partial C}{\partial q}(0)\right) .
$$

Then take $S(y)=(1 / 2) y^{T} H y$. 
We notice that the rank condition in condition (b) is weaker than the rank condition dim $\mathrm{d} \mathscr{G}_{0}(0,0)=2 n$ which we need for stabilization via linearization. (If $H=K+V$ has a strict local minimum in $(0,0)$ then the eigenvalues of the linearized system are all on the imaginary axis. Hence the linearized system has to be controllable in order to be stabilizable.).

One may wonder if condition (b) still cannot be relaxed, for instance by only requiring that $\operatorname{dim}^{C} \mathcal{C}(q, p)=2 n$ for $(q, p)$ in some subset, i.e. by only requiring some sort of strong accessibility or local weak observability (see theorem 1.1). However the following example shows this to be wrong.

Example. Consider the Hamiltonian system (1.1) with $Q=\mathbb{R}^{3}$.

$$
\begin{aligned}
& K=\frac{1}{2} p_{1}^{2}+\frac{1}{2} p_{2}^{2}+\frac{1}{2} p_{3}^{2}+\frac{1}{2} p_{1} p_{2} \sin q_{3}, \\
& V=\frac{1}{2} q_{1}^{2}+\frac{1}{2} q_{2}^{2}+\frac{1}{2} q_{3}^{2},
\end{aligned}
$$

and

$$
C_{1}(q)=q_{1}, C_{2}(q)=q_{2}
$$

Since $\left\{C_{1},\left\{H, C_{2}\right\}\right\}=-\frac{1}{2} \sin q_{3}, \operatorname{dim} \mathrm{d} \mathscr{G}(q, p)=6$ if $q \neq \frac{1}{2} \pi+k \pi, k \in \mathbb{Z}$, and the system is strongly accessible. However $\operatorname{dim} \mathrm{d} \mathscr{C}_{0}(q, p)=4$ everywhere and hence condition (b) is not satisfied. By applying the feedback $u_{1}=-c_{1} \dot{q}_{1}, u_{2}=-c_{2} \dot{q}_{2}$ we obtain the equations

$$
\begin{array}{ll}
\dot{q}_{1}=p_{1}+\frac{1}{2} p_{2} \sin q_{3} & \dot{p}_{1}=-q_{1}-c_{1} \dot{q}_{1} \\
\dot{q}_{2}=p_{2}+\frac{1}{2} p_{1} \sin q_{3} & \dot{p}_{2}=-q_{2}-c_{2} \dot{q}_{2} \\
\dot{q}_{3}=p_{3} & \dot{p}_{3}=-q_{3}-\frac{1}{2} p_{1} p_{2} \cos q_{3} .
\end{array}
$$

Hence $q_{1}=q_{2}=p_{1}=p_{2}=0$ is an invariant set, where the system equals the Hamiltonian system

$$
\begin{aligned}
& \dot{q}_{3}=p_{3} \\
& \dot{p}_{3}=-q_{3} .
\end{aligned}
$$

Therefore, the system is not asymptotically stable.

Remark. $\operatorname{dim} \mathrm{d} \mathscr{G}(q, p)=2 n$ also implies local weak observability. Therefore an alternative interpretation of the above example is that the input $u_{1}=u_{2}=0$ does not distinguish between any two states, and therefore is not universal (Sussmann [16]).

It follows from the proofs of theorem 2.1 and especially lemma 2.2 that under the assumption of theorem 2.1 condition (b) is unaffected by applying output feedback $u_{i}=-\left(\partial S / \partial y_{i}+v_{i}\right.$ with $\left(\partial S / \partial y_{i}\right)(C(0))=0$.

This constitutes a kind of analogue to the case of stabilization by linearization where the rank condition $\operatorname{dim} \mathscr{L}_{0}(0)=\operatorname{dim} M($ see (2.3)) is unaffected by applying feedback $u=\alpha(x, \bar{u})$ with $\alpha(0)=0$. It is an interesting question if this remains to be true if we drop the constant dimension assumption of theorem 2.1. 
For a comparison between stabilization by Lyapunov's first and second method for Hamiltonian systems we summarize the following points. A main advantage of the second method is the appealing form of the feedback $u_{i}=-k_{i} y_{i}-c_{i} \dot{y}_{i}$, i.e. linear output feedback of a decentralized nature which is physically interpretable (addition of potential energy and damping), while in the first method we need general state feedback. Another important advantage of the second method is that we can derive global stabilization results, or at least we can determine the regions of asymptotic stability. An advantage of the first method is that we can freely choose the eigenvalues of the linear part of the feedback transformed Hamiltonian vector field, if the linearized system is controllable. Hence we can make the system "as much asymptotically stable as we want", which is in the second method a more delicate issue (Tsinias and Kalouptsidis [10]; see however Jonckheere [11]).

The derived sufficient conditions for applying Lyapunov's first or second method are rather incomparable. Clearly, condition (b) is implied by the condition we need for controllability of the linearized system, since $\operatorname{dim} \mathrm{d}^{6} \mathscr{C}_{0}(0,0)=2 n$ implies that $\operatorname{dim} \mathrm{d} \mathscr{C}_{0}(q, p)=2 n$ in a neighborhood of $(0,0)$. However condition (a) does not follow from controllability of the linearized system. (It is easy to see that controllability only implies that the matrix

$$
\left(\frac{\partial^{2} V}{\partial q_{i} \partial q_{j}}(0)\right)
$$

is nonsingular restricted to

$$
\operatorname{Ker}\left(\frac{\partial C}{\partial q}(0)\right)
$$

hence we cannot use proposition 2.3.)

\section{AN APPLICATION TO ROBOT MANIPULATORS}

A general model for a robot manipulator is given by (Takegaki and Arimoto [14], Paul [17]):

$$
\begin{aligned}
& \dot{q}_{i}=\frac{\partial H}{\partial p_{i}} \\
& \dot{p}_{i}=-\frac{\partial H}{\partial q_{i}}+u_{i}
\end{aligned} \quad i=1, \ldots, n
$$

where $H(q, p)$ is the sum of a kinetic energy

$$
K(q, p)=\frac{1}{2} \sum_{i, j=1}^{n} g^{i j}(q) p_{i} p_{j}
$$

and a potential energy $V(q)$. Depending on the fact that if we are considering a translational or rotational joint $i$ the generalized coordinates $q_{i}$ are (joint) displacements or (joint) angles, and the generalized forces $u_{i}$ are translational external forces or external torques, which are delivered by actuators attached to each joint. The inverse matrix $\left(g_{i j}(q)\right)=\left(g^{i j}(q)\right)^{-1}$ appearing in the kinetic energy

$$
T(q, \dot{q})=\frac{1}{2} \sum_{i, j} g_{i j}(q) \dot{q}_{i} \dot{q}_{j}=K(q, p)
$$


is called the (effective) inertia matrix and the derivatives of $T$ w.r.t. the generalized coordinates

$$
\frac{1}{2} \sum_{i, j=1}^{n} \frac{\partial q_{i j}}{\partial q_{k}} \dot{q}_{i} \dot{q}_{i}
$$

yield the nonlinear interaction forces between the links, i.e. the centrifugal and Coriolis forces.

We notice that (3.1) constitutes a Hamiltonian system (1.1) with full observations $y=\left(y_{1}\right.$, $\left.\ldots, y_{n}\right)=\left(q_{1}, \ldots, q_{n}\right)$. Hence every generalized momentum variable $p_{i}$, and indirectly every $q_{i}$ can be controlled. Furthermore if we choose another set of output coordinates $\left(y_{1}^{i}, \ldots\right.$, $\left.y_{n}^{\prime}\right)=\phi\left(y_{1}, \ldots, y_{n}\right)$, for instance Cartesian coordinates instead of joint angles, then the generalized forces $\left(u_{1}, \ldots, u_{n}\right)$ are transformed to a set $\left(u_{1}^{\prime}, \ldots, u_{n}^{\prime}\right)$ given by

$$
\left(u_{1}^{\prime}, \ldots, u_{n}^{\prime}\right)=\left(u_{1}, \ldots, u_{n}\right)\left(\frac{\partial \phi_{i}}{\partial y_{j}}\left(y_{1}, \ldots, y_{n}\right)\right)^{-1} \text {. }
$$

In general these new generalized forces $\left(u_{1}^{\prime}, \ldots, u_{n}^{\prime}\right)$ are not anymore the forces as delivered by the actuators.

Since $\left(y_{1}, \ldots, y_{n}\right)=\left(q_{1}, \ldots, q_{n}\right)$ the conditions of theorem 2.1 are trivially satisfied and $\operatorname{dim} \mathrm{d} \mathfrak{G}_{0}(0,0)=2 n$. Furthermore it is easily seen that by output feedback $u_{i}=-\left(\partial S / \partial y_{i}\right)+v_{i}$ we can make every point $\bar{q}$ into a global minimum of the potential function $V+S$, if

$$
\left(\frac{\partial^{2} V}{\partial q_{i} \partial q_{j}}(q)\right)
$$

is bounded. In fact we may restrict ourselves to output feedback of the form (Takegaki and Arimoto [14]

$$
u_{i}=-\frac{\partial V}{\partial q_{i}}(\tilde{q})-k_{i}\left(q_{i}-\bar{q}_{i}\right)+v_{i}, \quad i=1, \ldots, n
$$

Hence by adding damping $v_{i}=-c_{i} \dot{q}_{i}, i=1, \ldots, n$, every point $(\bar{q}, 0)$ can be made globally asymptotically stable. Such a stabilization scheme can therefore be used for point-to-point control.

If not all generalized coordinates are available for observation it is clear that we cannot make every point $\bar{q}$ into a global minimum of the potential function $V+S$. However, for a point $\bar{q}$ that can be made a global minimum we may try to apply theorem 2.1 to stabilize the system around $(\bar{q}, 0)$.

Example. Consider the simple case (in Fig. 1) of two unit masses attached to the ends of links with length $l$. Suppose we observe the angle coordinate $y=q_{1}$ and we are able to exert an external torque $u$ around the first joint. We want to stabilize the system around $q_{1}=0$ and $q_{2}=\pi$.

The potential energy is given by (set $g=1$ )

$$
V=2 \cos q_{1}+\cos a_{2}
$$

and the kinetic energy by

$$
T=\dot{q}_{1}^{2}+\dot{q}_{2}^{2}+\dot{q}_{1} \dot{q}_{2} \cos \left(q_{1}-q_{2}\right)
$$


Hence

$$
\left(\frac{\partial^{2} V}{\partial q_{i} \partial q_{i}}(0, \pi)\right)=\left(\begin{array}{rr}
-2 & 0 \\
0 & 1
\end{array}\right) .
$$

Since this matrix is positive definite restricted to

$$
\operatorname{ker} \mathrm{d} C=\operatorname{span}\left(\begin{array}{l}
0 \\
1
\end{array}\right),
$$

there exists a feedback $u=-k y+v=-k q_{1}+v$ which makes $(0, \pi)$ into a local minimum of $\tilde{V}=V+k q_{1}^{2}$ (proposition 2.3). Indeed, take $k=2$, then $(0, \pi)$ is a global minimum of $\bar{V}$.

From the expression of $T$ it follows that

$$
\left(g^{i j}(0, \pi)\right)^{-1}=\left(\begin{array}{rr}
2 & -1 \\
-1 & 2
\end{array}\right)^{-1}=\frac{1}{3}\left(\begin{array}{ll}
2 & 1 \\
1 & 2
\end{array}\right) .
$$

It is now easy to check that the system linearized in $(0, \pi)$ is controllable and so dim $\mathrm{d}^{6} \mathscr{C}_{0}(0, \pi)$ is maximal $(=4)$. Hence by theorem 2.1 a damping $v=-c \dot{q}, c>0$, will make $(0, \pi)$ asymptotically stable.

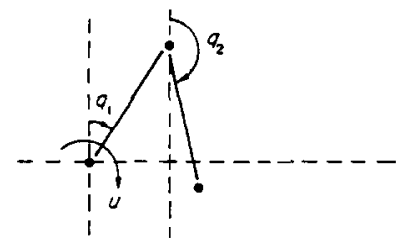

FiG. 1.

Even if we have full observations the stabilization scheme of Takegaki and Arimoto may be refined by using theorem 2.1 in such a way that we do not have to feed back every joint velocity $\dot{q}_{i}$ in order to achieve global asymptotic stability. In fact, suppose we want to make the point $(\bar{q}, 0)$ globally asymptotically stable by feedback of only one joint velocity, say $\dot{q}_{1}$. This can be accomplished in the following way.

$\left(1^{\circ}\right)$ Apply output feedback (3.3) such that

$$
\left(\frac{\partial^{2} V}{\partial q_{i} \partial q_{j}}(q)\right)+\left(\begin{array}{ll}
k_{1} & 0 \\
0 & \cdot k_{n}
\end{array}\right)>0
$$

for all $q$. Then the resulting potential funtion

has a global minimum in $q=\bar{q}$.

$$
\bar{V}=\frac{1}{2} \sum_{i=1}^{n} k_{i}\left(q_{i}-\bar{q}_{i}\right)^{2}
$$

$\left(2^{\circ}\right)$ Apply output feedback $v=R(q-\bar{q})+e_{1} \tilde{v}$, with $\tilde{v} \in \mathbb{R}$ and $R=R^{T}>0$, such that the linear Hamiltonian system

$$
\left(\begin{array}{l}
\dot{q} \\
\dot{p}
\end{array}\right)=\left(\begin{array}{cc}
0 & P \\
-Q & 0
\end{array}\right)\left(\begin{array}{l}
q \\
p
\end{array}\right)+\left(\begin{array}{l}
0 \\
e_{1}
\end{array}\right) \bar{v}
$$


with

$$
P=\left(g^{i j}(\bar{q})\right), \quad Q=\left(\frac{\partial^{2} \bar{V}}{\partial q_{l} \partial q_{j}}(\bar{q})\right)+R,
$$

and $e_{1}$ the first basis vector in $\mathbb{R}^{n}$; is controllable. (Since the linearized system is controllable, there always exists such an $R$.)

$\left(3^{\circ}\right)$ Finally apply the feedback $\bar{v}=-c \dot{q}_{1}, c>0$. Since by $\left(2^{\circ}\right)$, condition (b) of theorem 2.1 is satisfied with respect to only one output function $y_{1}=C_{1}\left(q_{1}\right)=q_{1}$, the resulting system is globally asymptotically stable.

In general the matrix $R$ in $\left(2^{\circ}\right)$ has to contain nonzero off-diagonal elements. Hence the price we have to pay for stabilization by feeding back only one joint velocity is that we have to use a static output feedback with respect to the generalized coordinates $\left(y_{1}, \ldots y_{n}\right)=\left(q_{1}\right.$, $\left.\ldots, q_{n}\right)$, which is not of a decentralized nature and introduces extra couplings between the links.

\section{CONCLUSION}

In [11] and [14] it has been shown how a Hamiltonian system with a number of independent external forces which equals the degree of freedom of the system, can be stabilized using Lyapunov's second method. In this paper we extend this approach to the case that one is limited to a smaller number of external forces and to the measurement of only a part of the generalized coordinates and velocities. This also constitutes a refinement of some related results independently obtained by Tsinias and Kalouptsidis [10] and Marino [12]. The resulting feedback scheme is from a physical point of view very appealing, and gives rigor to the intuitive idea that damping in a part of the system may cause stability of the whole system. From a mathematical point of view it would be interesting to make a closer study of the space $\mathscr{C}_{0}$ and its zero-set $N_{0}$, especially in connection with the structure of the Lie algebra $b$ determining the "controllability and observability" of the system.

Acknowledgements-I would like to thank H. Th. Jongen and H. Nijmeijer for some useful discussions.

\section{REFERENCES}

1. SCHAFT A. J. VAN DER, System theoretic descriptions of physical systems, Doctoral Dissertation, University of Groningen; CWI Tracts No. 3, CWI, Amsterdam (1984).

2. BRoCKETT R. W., Control theory and analytical mechanics, in Geometric Control Theory (Edited by C. MARTIN and R. Hermanis), Vol VII, pp. 1-46, Lie Groups, History, Frontiers and Applications, Math. Sci. Press, Brookline (1977).

3. SCHAFT A. J. VAN DER, Hamiltonian dynamics with external forces and observations, Math. Systems Theor. 15. $145-168$ (1982).

4. SCHAFT A. J. VAN DER, Symmetries, conservation laws and time reversibility for Hamiltonian systems with external forces, J. Math. Phys. 24, 2095-2101 (1983).

5. SpIvaK M., A Comprehensive Introduction to Differential Geometry, Vol. I. Publish or Perish, Boston (1970).

6. SCHAFT A. J, VAN DER, Controllability and observability for affine nonlinear Hamiltonian systems. IEEE Trans. Automat. Control AC-27, 490-492 (1982).

7. SCHAFT A. J. VAN DER, Symmetries and conservation laws for Hamiltonian systems with inputs and outputs: A generalization of Noether's theorem, Systems Control Letts, 1, 108-115 (1981).

8. LEE E. B. \& MARkus L., Foundations of Optimal Control Theory, Wiley, New York (1976).

9. JlRdieviC V. \& Quiviv J. P., Controllability and Stability, J. diff. Eqns 28, 381-389 (1978).

10. Tsinias J. \& Kaloupsidis N., On stabilizability of nonlinear systems, Proc. 21st IEEE Conf. on Decision and Control, pp. 712-716 (1982).

11. JONCKHEERE E. A., Lagrangian theory of large scale systems, manuscript, University of Southern California, Department of Electrical Engineering Systems (1981). 
12. Marino R., Stabilization and feedback equivalence to linear coupled oscillators, Int. J. Control 39, 487-496 (1984).

13. La Salle J. P. \& Lefschetz S., Stability by Liapunov's Direct Method with Applications, Academic Press, New York (1961).

14. TAKEGAKI M. \& ARIMOTO S., A new feedback method for dynamic control of manipulators, Trans. ASME, J. Dyn. Systems, Meas. Control 103, 119-125 (1981).

15. JoNGeN H. Th., JONKER P. \& TWILT F., Nonlinear Optimization in $\mathrm{R}^{n}$, I: Morse Theory, Chebyshev Approximation, Peter Lang, Frankfurt a. M. (1983).

16. SussmaNN H. J., Single-input observability of continuous-time systems, Math. Systems Theor. 12, 371-393 (1979).

17. PAUL R. P., Robot Manipulators: Mathematics, Programmings, and Control, MIT Press, Cambridge, Mass. (1981). 